\title{
Disrupted dynamic functional network connectivity among cognitive control networks in the progression of Alzheimer's disease
}

\author{
Mohammad S. E. Sendi ${ }^{1,2,3}$, Elaheh Zendehrouh ${ }^{4}$, Zening $\mathrm{Fu}^{3}$, Jingyu Liu ${ }^{3,4}$, Yuhui $\mathrm{Du}^{3,5}$, \\ Elizabeth Mormino $^{6}$, David H. Salat ${ }^{7,8}$, Vince D. Calhoun ${ }^{1,2,3,4_{\uparrow}}$, Robyn. L. Miller ${ }^{3,4_{\dagger}}$
}

${ }^{1}$ Wallace H. Coulter Department of Biomedical Engineering at Georgia Institute of Technology and Emory University, Atlanta, GA; ${ }^{2}$ Department of Electrical and Computer Engineering at

Georgia Institute of Technology, Atlanta, GA; ${ }^{3}$ Tri-institutional Center for Translational Research in Neuroimaging and Data Science, Georgia State University, Georgia Institute of Technology, Emory University, Atlanta, GA; ${ }^{4}$ Department of Computer Science at Georgia State University, Atlanta, GA; ${ }^{5}$ School of Computer and Information Technology, Shanxi University,

Taiyuan, China; ${ }^{6}$ Stanford Medical School, Palo Alto, CA, ${ }^{7}$ Harvard Medical School, Cambridge, MA, ${ }^{8}$ Massachusetts General Hospital, Boston, MA E-mail: vcalhoun@gsu.edu (Corresponding author: Vince D. Calhoun) $\dagger$ Equal contribution as senior authors 


\begin{abstract}
:
Background: Alzheimer's disease (AD) is the most common age-related dementia that promotes a decline in memory, thinking, and social skills. The initial stages of dementia can be associated with mild symptoms, and symptom progression to a more severe state is heterogeneous across patients. Recent work has demonstrated the potential for functional network mapping to assist in the prediction of symptomatic progression. However, this work has primarily used static functional connectivity (sFC) from rs-fMRI. Recently, dynamic functional connectivity (dFC) has been recognized as a powerful advance in functional connectivity methodology to differentiate brain network dynamics between healthy and diseased populations.
\end{abstract}

Methods: Group independent component analysis was applied to extract 17 components within the cognitive control network $(\mathrm{CCN})$ from 1385 individuals across varying stages of $\mathrm{AD}$ symptomology. We estimated dFC among 17 components within the $\mathrm{CCN}$, followed by clustering the $\mathrm{dFCs}$ into 3 recurring brain states and then estimated a hidden Markov model and the occupancy rate for each subject. Finally, we investigated the link between CCN dFC connectivity features with $\mathrm{AD}$ progression.

Results: Progression of AD symptoms were associated with increases in connectivity within the middle frontal gyrus. Also, the AD with mild and severer symptoms showed less connectivity within the inferior parietal lobule and between this region with the rest of CCN. Finally, comparing with mild dementia, we found that the normal brain spends significantly more time in a state with lower within middle frontal gyrus connectivity and higher connectivity between the hippocampus and the rest of $\mathrm{CCN}$, highlighting the importance of assessing the dynamics of brain connectivity in this disease.

Conclusion: Our results suggest that $\mathrm{AD}$ progress not only alters the $\mathrm{CCN}$ connectivity strength but also changes the temporal properties in this brain network. This suggests the temporal and spatial pattern of $\mathrm{CCN}$ as a biomarker that differentiates different stages of AD.

Impact Statement: By assuming that functional connectivity is static over time, many of previous studies have ignored the brain dynamic in Alzheimer's disease progression. Here, a longitudinal resting-state functional magnetic resonance imaging data are used to explore the temporal changes of functional connectivity in the cognitive control network in Alzheimer's disease progression. The result of this study would increase our understanding about the underlying mechanisms of Alzheimer's Disease and help in finding future treatment of this neurological disorder.

Key words: Alzheimer's disease, cognitive control network, dynamic functional connectivity, resting-state fMRI 


$\begin{array}{ll}\text { Abbreviations } \\ \text { AD } & \text { Alzheimer's disease } \\ \text { vmAD } & \text { very mild Alzheimer's disease } \\ \text { dFC } & \text { Dynamic functional connectivity } \\ \text { sFC } & \text { Static functional connectivity } \\ \text { MCI } & \text { Mild cognitive impairment } \\ \text { CCN } & \text { Cognitive control network } \\ \text { OCR } & \text { Occupancy rate } \\ \text { CDR-SOB } & \text { Clinical dementia rating scale sum of boxes } \\ \text { HC } & \text { Healthy control } \\ \text { EPI } & \text { Echo-planar imaging } \\ \text { MNI } & \text { Montreal Neurological Institute } \\ \text { FWHM } & \text { Full width at half maximum } \\ \text { MMSE } & \text { Mini-mental state examination } \\ \text { ICA } & \text { Independent component analysis } \\ \text { IC } & \text { Independent component }\end{array}$

\section{Introduction}

Alzheimer's disease (AD) is the most common age-related dementia, which affects 10-30\% of individuals over 65 years of age (Masters et al., 2015). It usually promotes a decline in memory, cognition, everyday function, and social skills. AD usually progresses slowly in 3 stages, including mild cognitive impairment (early-stage), mild dementia (middle-stage), and severe dementia (latestage) (Ryan and Rossor, 2011). To date, there is no treatment for AD, but some interventions, including pharmacological (Massoud and Léger, 2011) and non-pharmacological (Shigihara et al., 2020; Zucchella et al., 2018) can decelerate its progress in particular when it is detected at an early stage(Yiannopoulou and Papageorgiou, 2020). Predicting AD progression and differentiating different stages of this disease are thus essential steps in early medical intervention for this mental disorder (Badhwar et al., 2017; Brand et al., 2019; Gupta et al., 2019; Kruthika et al., 2019; Lee, Garam, 2019; Z. Wang et al., 2019). Also, knowing that the initial stages of dementia show heterogeneous symptoms across patients, identifying individuals at risk for progression from mild cognitive impairment (MCI) to early or late dementia is challenging (Komarova and Thalhauser, 2011).

In recent years, functional connectivity (FC) obtained from resting-state functional magnetic resonance imaging (rs-fMRI) has shown sensitivity to the prediction of current and future AD 
based on brain network connectivity (Badhwar et al., 2017; Brier et al., 2014; Wang et al., 2007 ; Z. Wang et al., 2019; Zhao et al., 2020). A large body of the AD-related literature has focused on default mode network (DMN) FC disruption, and other brain networks are underexplored (Agosta et al., 2012; Balthazar et al., 2014; Miao et al., 2011; Zhong et al., 2014). But other brain networks such as the cognitive control network $(\mathrm{CCN})$ are rarely investigated in $\mathrm{AD}$ progression. As previous studies showed, CCNwhich includes inferior parietal lobule, inferior frontal gyrus, middle frontal gyrus, hippocampus, insula, middle cingula cortex, and superior frontal gyrus, is involved in executive function including working memory, internal and external navigation, and attention (Breukelaar et al., 2017; Dhanjal and Wise, 2014; Han et al., 2018; Y. Wang et al., 2019; Westerhausen et al., 2010), which are impaired in AD (Masters et al., 2015). For example, a recent study showed that $\mathrm{AD}$ patients have more activation in superior frontal gyrus, middle frontal gyrus, and parietal region compared with normal subjects during a working memory task (Yetkin et al., 2006). Another one found changes in the inferior parietal lobule thickness by progression from normal to MCI (Greene and Killiany, 2010). Another study showed a disconnection between the hippocampus and other brain regions in $\mathrm{AD}$ patients (Allen et al., 2007). Therefore, we hypothesized that studying this brain network would reveal useful information about the fundamental neuronal mechanism of $\mathrm{AD}$ and its longitudinal progression.

Unlike conventional static FC (sFC), which represents the averaged brain connections over an entire scan, dynamic FC (dFC) refers to brain connectivity within sub-intervals of the time series (Calhoun et al., 2014). In recent years, dFC from rs_fMRI time series has proven highly informative regarding the underlying brain connectivity patterns in different neurological disorders, including schizophrenia (Dong et al., 2019; Sendi et al., 2020a, 2020b), major depressive disorder (Zendehrouh et al., 2020), and AD (Fiorenzato et al., 2019; Fu et al., 2019). Furthermore, features obtained from $\mathrm{dFC}$ are shown to be more sensitive to brain disorders, for example, in classifying healthy subjects from patients, than their sFC counterparts (Rashid et al., 2016; Vergara et al., 2018). Any cognitive deficits and clinical symptoms associated with brain disorders likely depend not only on the strength of the connectivity between any pair of the specific brain regions but also on the patterns of temporal variation in the connectivity of those regions.

In this work, from longitudinal rs-fMRI data (LaMontagne et al., 2019), we predicted that CCN $\mathrm{dFC}$ in $\mathrm{AD}$ and its correlation with behavioral scores could elucidate the pathophysiological mechanism of this neurological disorder. More specifically, we hypothesized that cognitive states 
in different stages of $\mathrm{AD}$ could be linked to the temporal fluctuations exhibited by $\mathrm{CCN} \mathrm{dFC}$ in this group of subjects. To find this link, we leveraged the sliding window approach followed by kmeans clustering to identify a set of connectivity states to investigate $\mathrm{dFC}$ within the $\mathrm{CCN}$ (Allen et al., 2014). We explored the link between symptom severity in AD with state-specific CCN FC. To further investigate and model the temporal changes in $\mathrm{dFC}$, we estimated the occupancy rate (OCR) for each subject from dFC. Next, we explored the link between these features and cognitive scores via statistical analysis on the estimated OCR features.

\section{Methods}

In this study, ethical approval was granted by the relevant ethics committees, and informed consent was obtained from each subject prior to scanning according to the institutional review board of Washington University School of Medicine.

\section{Participant}

The data is from the Open Access Series of Imaging Studies (OASIS)-3 cohort, which contains imaging and related clinical data of 1098 participants. The data are collected across several ongoing studies in the Washington University Knight Alzheimer Disease Research Center over 15 years. This study used 1385 rs-fMRI and clinical and demographic information at scanning (from 910 subjects) with the age range of 42 to 95 years (LaMontagne et al., 2019). The clinical dementia rating scale sum of boxes (CDR-SOB) scores is used to assess the cognitive stage of the participant at the time of scanning. All subjects must have $\mathrm{CDR} \leq 1$ at the time of the clinical core assessment and once the participant reached $\mathrm{CDR}=2$ or $\mathrm{CDR}-\mathrm{SOB}>9$, they were no longer eligible for the study (LaMontagne et al., 2019). Using CDR-SOB, we categorized all subjects into two groups, including healthy control or $\mathrm{HC}(\mathrm{CDR}-\mathrm{SOB}=0)$, and very mild $\mathrm{AD}$ or vmAD (CDR-SOB>0) (O’Bryant et al., 2008). In total, we had 1028 scans of HC, 357 scans of vmAD patients. The demographic information is provided in Table 1.

\section{Data Acquisition}

MRI data were collected from two scanners of TIM Trio 3T with a 20-channel head coil and foam pad stabilizers placed next to the ears to decrease motion (Siemens Medical Solutions USA, Inc). 
High resolution $\mathrm{T} 2 *$-weighted functional images were acquired using echoplanar imaging or EP sequence with $\mathrm{TE}=27 \mathrm{~ms}, \mathrm{TR}=2.2 \mathrm{~s}$, flip angle $=90^{\circ}$, slice thickness $=4 \mathrm{~mm}$, slice gap (centerto-center $)=4 \mathrm{~mm}$, matrix size $=64$, and field of view $(\mathrm{FOV})=256 \times 256 \times 128 \mathrm{~mm}^{3}$. The duration of the scanning was 6 minutes.

\section{Data processing}

We processed the fMRI data using statistical parametric mapping (SPM12, https://www.fil.ion.ucl.ac.uk/spm/) in the MATLAB2019 environment. The first five dummy scans were discarded before preprocessing, and a slice-timing correction was performed on the fMRI data. To account for the subject's head motion, we used rigid body motion correction. Next, the imaging data underwent spatial normalization to echo-planar imaging (EPI) template in the standard Montreal Neurological Institute (MNI) space and was resampled to $3 \times 3 \times 3 \mathrm{~mm}^{3}$. Finally, we used a Gaussian kernel to smooth the fMRI images using a full width at half maximum (FWHM) of $6 \mathrm{~mm}$.

We used the Neuromark automated independent component analysis (ICA) pipeline, which uses previously derived component (region) maps as priors for spatially constrained ICA (Du et al., 2019), to extract reliable CCN independent components (ICs) or components. In Neuromark, replicable components were identified by matching group-level spatial maps from two largesample healthy control (HC) datasets. Components were identified as meaningful regions if they exhibited peak activations in the gray matter within CCN. Seventeen data-driven CCN components were identified. These components are shown in Table 2.

\section{Dynamic functional connectivity (dFC)}

For each subject, $\mathrm{i}=1 \ldots \mathrm{N}$, the $\mathrm{dFC}$ of the $17 \mathrm{CCN}$ components was estimated via a sliding window approach, as shown in Fig. 1. A tapered window obtained by convolving a rectangle (window size $=20 \mathrm{TRs}=44 \mathrm{~s})$ with a Gaussian $(\sigma=3 \mathrm{~s})$ was used to localize the dataset at each time point. A correlation matrix, based on Pearson correlation, was calculated to measure the $\mathrm{dFC}$ between CCN components (Step 1 in Fig. 1). The dFC estimates of each window for each subject were concatenated to form a $(\mathrm{C} \times \mathrm{C} \times \mathrm{T})$ array (where $\mathrm{C}=17$ denotes the number of components and $\mathrm{T}=139$ windows), which represented the changes in brain connectivity between $\mathrm{CCN}$ 
components as a function of time (Calhoun et al., 2014).

\section{Clustering and Latent Feature Estimation}

A k-means algorithm was applied to the $\mathrm{dFC}$ windows to partition the data into a set of separated clusters. The optimal number of centroid states was estimated to be 3 using the elbow criterion based on the ratio of within to between cluster distance. This optimal number was found by searching between 2 to 9 . We used the Euclidean distance metric in the clustering algorithm with 1000 iterations (Allen et al., 2014; Calhoun et al., 2014). The clustering output was 3 states for all subjects and state vector for each subject (Step 2 in Fig. 1). Next, to model the temporal changes of $\mathrm{dFC}$ for each subject using the state vector, we computed the OCR of dFCs in each state. OCR represents the proportion of time each subject occupies a given state. (Step3 in Fig. 1). From 3 states, we calculated 3 OCR features for each subject.

\section{Statistical Analysis}

To find a link between $\mathrm{dFC}$ features, including OCR, and the cell values of the $\mathrm{dFC}$ in each state with mini-mental state examination (MMSE) score, we used partial correlation accounting for age and gender. We performed statistical analysis on the link between 136 state-specific CCN features obtained from $17 \mathrm{CCN}$ components of each subject and clinical score, and between 3 OCR features obtained from state vectors of each subject and the clinical score, separately. All p values (3 pvalues for the OCR and $136 \mathrm{p}$-values for state-specific CCN features with clinical score) have been adjusted by the Benjamini-Hochberg method for false discovery rate or FDR (Yoav Benjamini $\square$; Yosef Hochberg, 1995).

\section{Results}

\section{Clinical and demographic results}

The mean CDR-SOB of HC and vmAD were $0 \pm 0,2.59 \pm 1.92$, respectively. The mean MMSE of $\mathrm{HC}$ and vmAD were $28.88 \pm 1.34,25.94 \pm 3.60$, respectively. Using a two-sample t-test, we found significant differences in MMSE between $\mathrm{HC}$ and $\operatorname{vmAD}\left(\mathrm{p}<1 \mathrm{e}^{-5}\right)$. The mean age of $\mathrm{HC}$ and 
vmAD were $69.83 \pm 8.64$ and $75.18 \pm 7.77$, respectively. Using the two-sample t-test, we found significant differences between $\mathrm{HC}$ and vmAD age $\left(\mathrm{t}(1384)=-10.62\right.$, uncorrected $\left.\mathrm{p}=3.91 \mathrm{e}^{-24}\right)$. It worth noticing that due to the significant age differences among the groups, we controlled the age and gender in all correlation analysis we did in this study.

\section{Overview of dFC states}

Fig. 2 shows the 3 reoccurring dFC states, including state 1, state 2, and state 3 identified by kmeans clustering. In all states, FCs within inferior parietal lobule, inferior frontal gyrus, the middle frontal gyrus, and the hippocampus were positive. In all states, among all subregions of CCN, the hippocampus had the lowest connectivity with the rest of CCN. Also, among all states, state 1 showed the strongest negative connectivity between the hippocampus and the rest of CCN. In addition, state 2 showed the least within the middle frontal gyrus comparing with that of other states. This state relatively showed more connectivity between hippocampus and the rest of CCN compared with that of other states. Also, state 3 showed the strongest positive connectivity within the inferior frontal gyrus. Finally, we measured the OCR of each subject in state1, state2, and state 3. OCR represents the amount of time each subject spends in each state. We found subjects spend $33.68 \%, 28.02 \%$, and $38.30 \%$ in state 1 , state 2 , and state 3, respectively. In addition, using a paired t-test, we found a significant difference between the OCR of state 1 and state $2(t(1384)=$ 4.62, FDR corrected $\left.<1 \mathrm{e}^{-4}\right)$ and between state 1 and state $3\left(\mathrm{t}(1384)=3.73\right.$, FDR corrected $\left.\mathrm{p}<1 \mathrm{e}^{-4}\right)$, and between state 2 and state $3\left(\mathrm{t}(1384)=8.62\right.$, FDR corrected $\left.\mathrm{p}<1 \mathrm{e}^{-4}\right)$.

\section{Correlation between dFC cell features and MMSE}

In each state, we averaged the connectivity features of all $\mathrm{dFC}$ for each subject. In more detail, each subject has multiple $\mathrm{dFC}$ in each state. Then, in each state, we used the average of $\mathrm{dFC}$ features (i.e., the average of 136 connectivity features) of each subject as her/his state-specific FC. Then, we calculated the partial correlation between averaged cell features of each subject and MMSE by controlling the age and gender to explore how these features changed by progressing from HC stages to AD stages. These results are shown in Fig. 3. The promising correlations (FDR uncorrected $\mathrm{p}<0.05)$ are shown in red (positive correlation) and blue (negative correlation). Also, a significant correlation that passes the FDR correction is marked by asterisks. 
In state1, we observed a positive correlation between MMSE and the connectivity of inferior parietal lobule connection with inferior frontal gyrus. Also, we found a negative correlation between MMSE and the connectivity within the middle frontal gyrus. In state 2, we found that within middle frontal gyrus connectivity and its connectivity with inferior frontal gyrus showed a negative link with MMSE. In state 3, we found that the connection between inferior frontal lobule and the rest of $\mathrm{CCN}$, including inferior frontal gyrus, middle frontal gyrus, insula, superior frontal gyrus, and middle cingula cortex showed a positive correlation with MMSE. Similar to state 1 and state 2 , we observed a negative link between within middle frontal gyrus connectivity and MMSE.

\section{Correlation between dFC temporal pattern and MMSE}

To find a link between MMSE and temporal features of $\mathrm{dFC}$, including OCR, we used partial correlation by controlling age and gender. By calculating the correlation between MMSE and OCR, we found the OCR of state2, which representing spending time in state 2 , shows a positive correlation with MMSE (Fig. 5: $\mathrm{r}=0.073$, FDR corrected $\mathrm{p}=0.01, \mathrm{n}=1385$ ). This means subjects with more severe symptoms spent less time in state 2 , which showed the least middle frontal gyrus connectivity.

\section{Discussion}

Recent studies proved that the brain functional connectivity obtained from rs-fMRI is exceedingly dynamic and can disclose the underlying mechanism differences of brain connectivity in many diseases groups (Damaraju et al., 2014; Dong et al., 2019; Fiorenzato et al., 2019; Fu et al., 2019; Garrity et al., 2007; Miller et al., 2016; Sun et al., 2019; Zhi et al., 2018). In addition, it seems that the cognitive control network $(\mathrm{CNN})$, which plays a significant role in several cognitive functions, degrades as AD progress (Dhanjal and Wise, 2014). Therefore, in the current study, we posit that studying the spatiotemporal pattern of $\mathrm{CCN}$ functional connectivity would add new information about the progression of $\mathrm{AD}$. In more detail, we investigated the $\mathrm{dFC}$ of several data-driven components of $\mathrm{CCN}$, including inferior parietal lobule, inferior frontal gyrus, middle frontal gyrus, hippocampus, insula, middle cingula cortex, and superior frontal gyrus. We found that functional connectivity in $\mathrm{CCN}$ is indeed highly dynamic, representing flexibility in functional coordination in this mode. Then, in each state, we used a partial correlation between FC features 
and MMSE to explore whether the FC features show a link with cognitive scores or not.

We found that the connectivity of the inferior parietal lobule with other regions of $\mathrm{CCN}$ has a positive correlation with MMSE in state 3, which means by progression from the normal state to $\mathrm{AD}$ state, the $\mathrm{FC}$ of the inferior parietal lobule with other regions decreases. Some evidence from neuroimaging studies implies the role of the inferior parietal lobule in a broad range of behavior and function, including cognitive functionality (Bzdok et al., 2016; Caspers et al., 2013; Wang et al., 2017). For example, a previous study proved the vital role of an inferior parietal region in maintaining attentive control (Shapiro and Hillstrom, 2002). A reduction of gray matter volume in the inferior parietal lobule by the progression of AD is reported (Greene and Killiany, 2010; Oishi et al., 2018). A recent research paper reported an improvement of working memory in AD patients by applying transcranial direct current stimulation to the inferior parietal region (Roncero et al., 2017). All these studies emphasized the role of this region in AD progression. However, inferior parietal lobule functional connectivity is understudied.

In our study, using a relatively large dataset, we found that inferior parietal lobule functional connectivity is affected as $\mathrm{AD}$ progresses, more so than other regions of CNN studied here, as well as in the early stage of $\mathrm{AD}$ by progression from the normal brain to $\mathrm{AD}$. Therefore, this region could be a reasonable brain area to target for future AD studies. However, this connectivity does not show a significant correlation with MMSE in state 2. This aberrant spatiotemporal pattern in the connectivity of inferior parietal lobule and CCN subregions potentially underlined the importance of studying the $\mathrm{dFC}$ and analyzing the $\mathrm{FC}$ in a shorter period of time. A recent study showed a disrupted pattern between the inferior parietal lobule and default mode, salience, executive control, and sensorimotor networks (Wang et al., 2015). The current study provides new knowledge about the disrupted pattern between the inferior parietal lobule and the rest of CCN.

The inferior frontal gyrus also showed a disrupted pattern. Although we found that the connectivity between inferior frontal gyrus and inferior parietal lobule in state 1 and state 3 decreases by progression from healthy to $\mathrm{AD}$, the connectivity between inferior frontal gyrus and middle frontal gyrus of state 2 increases in this progression. In a pilot study, the inferior frontal gyrus was stimulated by transcranial magnetic stimulation for improving attention in the early stage of AD. However, the result was not consistent and significant in all tests (Eliasova et al., 2014). Our result of having multiple distinct patterns in the inferior frontal gyrus connectivity further highlights the importance of additional studies evaluating the potential of $\mathrm{AD}$ intervention 
by modulating these connectivity patterns.

Interestingly, we found that the increase within middle frontal gyrus connectivity positively correlates with cognition decline in $\mathrm{AD}$ with mild symptoms. A previous study introduced a compensatory mechanism of neural resources in $\mathrm{AD}$ by showing more activation of the middle frontal gyrus in AD patients introduced as (Woodard et al., 1998). In the current study, a higher middle frontal gyrus connectivity in $\mathrm{AD}$ would further support the compensatory mechanism to reduce the impact of the decrease of connectivity in other regions of $\mathrm{CCN}$ in $\mathrm{AD}$ subjects (Gaubert et al., 2019). Also, like the inferior parietal lobule and inferior frontal gyrus, we observed a disrupted pattern in the connectivity between the middle frontal gyrus and CCN subregions. Interestingly, the hippocampus is showing the least effect among all regions in the CCN. This result is consistent with a previous study showing that the hippocampus connectivity shows fewer changes in early-onset AD (Park et al., 2017). Compared with other areas in CCN, these pieces of evidence might suggest that hippocampus connectivity with other subregions of $\mathrm{CCN}$ is one of the last brain areas affected by AD. However, a previous study found a dysconnectivity between the hippocampus and posterior cingulate cortex (PCC) as a part of DMN (Grieder et al., 2018). Therefore, our finding might be limited to the connectivity between hippocampus and $\mathrm{CCN}$ subregions, and the connectivity between the hippocampus and other brain networks beyond $\mathrm{CCN}$, might be disrupted with AD progression.

Next, to model the temporal pattern of $\mathrm{dFC}$, we estimated the OCR. Also, to explore how the temporal pattern of $\mathrm{dFC}$ correlates with cognition, we calculated the correlation between OCR and MMSE by controlling age and gender. In this analysis, we found dwell time of state 2 showed a significant and positive correlation MMSE. This means that subjects participants with mild impairment and mild symptoms tend to stay less in a state, which showed the least connectivity within the middle frontal gyrus among all states, than a normal brain. This provides further evidence of the effect of disease on the dysregulating temporal properties of CCN FC.

In addition, state 2 showed relatively higher connectivity between the hippocampus and the rest of the brain. Although we did not find any significant correlation between hippocampus connectivity strength and clinical rate, the temporal dysregulation effect of the $\mathrm{AD}$ progression might reveal some new information about the effect of this disease on the hippocampus, even in the early stage of the disease. This finding potentially highlighted the importance of the study of functional connectivity in a shorter period. Previous studies highlighted the effect of AD on the temporal 
pattern on brain FC. One study with $29 \mathrm{AD}$ patients and $31 \mathrm{HC}$ subjects found that $\mathrm{AD}$ patients spend more time than the $\mathrm{HC}$ subject in a state with sparse connectivity pattern in which the motor network is isolated from the rest of the brain. In addition, the same study found an inability to switch out from a state with low inter-network connectivity into more highly connected network configurations in $\mathrm{AD}$ patients (Schumacher et al., 2019) . In another study, an altered dFC temporal pattern has been shown in Parkinson's disease with dementia, consistent with the result of the current study (Fiorenzato et al., 2019). In the current study, we provided new evidence about the effect of $\mathrm{AD}$ progression on altering the temporal pattern of $\mathrm{CCN}$.

\section{Limitations and Future directions}

There are some limitations to this work. MMSE is commonly used to measure the cognitive state of the brain in the different stages of AD. However, a few studies suggested that MMSE is a noisy measure in the diagnosis of AD (Wang et al., 2015). The choice of window size is an implicit assumption about the dynamic behavior in that a short window captures more rapid fluctuations, whereas a longer window does more smoothing than a shorter one. Previous $\mathrm{dFC}$ studies showed that the window size between 30-60 $\mathrm{s}$ is a reasonable choice for capturing dFC fluctuation and any widow size above the safety limit, i.e., the largest wavelength present in the preprocessed fMRI time courses, would not change the result significantly (Preti et al., 2017). In addition, k-means clustering needs predefined knowledge for setting the clustering parameters, including the distance metrics. By applying different distance metrics, we did not observe a significant difference in the results, however applying other clustering methods such as robust continuous clustering would eliminate this shortcoming and might improve the clustering results (Shah and Koltun, 2017).

As mentioned earlier, a previous study showed a dysconnectivity between the hippocampus as a part of CCN and DMN in AD (Grieder et al., 2018). Therefore, a prospective study on the effect of this dysconnectivity between $\mathrm{CCN}$ and DMN, and its links with AD progression is needed. Although in the current study we focused on the CCN based on the prior knowledge of its role in cognitive impairment, future studies and methods that can mechanistically remove the irrelevant networks in study dynamic functional connectivity are needed (Cohen et al., 2015; Qiao et al., 2019; Schlesinger et al., 2017).

\section{Conclusion}


To summarize, we explored the alteration of temporal features of CCN FC by comparing participants of the normal state, mild impairment, and mild dementia. We found that $\mathrm{AD}$ progression affects differently on CCN subregions. It decreases inferior parietal lobule connectivity with other regions and increases within- middle frontal gyrus connectivity. We found both decrease and increase in inferior frontal gyrus and middle frontal gyrus connectivity with the rest of $\mathrm{CCN}$ subregions. Also, we found by progression from normal to mild dementia stage decreases the dwell time in a state with less within middle frontal gyrus and higher connectivity between the hippocampus and rest of the $\mathrm{CCN}$. This result further supports the role of the middle frontal gyrus in the progression of AD. Interestingly, while local (cell-wise) hippocampal patterns were not impacted by $\mathrm{AD}$ progression, more global (state-based) patterns linking $\mathrm{CCN}$ to hippocampus showed lower occupancy as AD progressed. Our results suggest that AD progress changes not only the connectivity strength but also affects the temporal properties in the brain network connectivity. In other words, our finding posits the temporal and spatial pattern of CCN as a biomarker in $\mathrm{AD}$ that differentiates patients based on symptom severity.

\section{Author Contribution}

Mohammad S. E. Sendi developed the study, conducted data analysis, interpreted the results, and wrote the original manuscript draft. Elaheh Zendehrouh conducted data analysis. Zening $\mathrm{Fu}$ conducted data analysis. Jingyu Liu edited the original draft and provided critical review to the initial draft. Yuhui Du developed the Neuromark and provided critical review to the initial draft. Elizabeth Mormino provided critical review to the initial draft. David H. Salat edited the original draft and provided critical review to the initial draft. Vince D. Calhoun developed the study, interpreted the results, edited the original draft, and provided critical review to the initial draft. Robyn L. Miller developed the study, interpreted the results, edited the original draft, and provided critical review to the initial draft. All authors approved the final manuscript.

\section{Author Disclosure Statement}

No competing financial interests exist.

\section{Funding Information}


The following NIH grants funded this work: R01AG063153, R01EB020407, R01MH094524,

R01MH119069, R01MH118695, and R01MH121101.

\section{Acknowledgement}

We thank those who helped collect this valuable data.

\section{References}

Agosta, F., Pievani, M., Geroldi, C., Copetti, M., Frisoni, G.B., Filippi, M., 2012. Resting state fMRI in Alzheimer's disease: Beyond the default mode network. Neurobiology of Aging 33, 1564-1578. https://doi.org/10.1016/j.neurobiolaging.2011.06.007

Allen, E.A., Damaraju, E., Plis, S.M., Erhardt, E.B., Eichele, T., Calhoun, V.D., 2014. Tracking whole-brain connectivity dynamics in the resting state. Cerebral Cortex 24, 663-676. https://doi.org/10.1093/cercor/bhs352

Allen, G., Barnard, H., McColl, R., Hester, A.L., Fields, J.A., Weiner, M.F., Ringe, W.K., Lipton, A.M., Brooker, M., McDonald, E., Rubin, C.D., Cullum, C.M., 2007. Reduced hippocampal functional connectivity in Alzheimer disease. Archives of Neurology 64, 1482-1487. https://doi.org/10.1001/archneur.64.10.1482

Badhwar, A.P., Tam, A., Dansereau, C., Orban, P., Hoffstaedter, F., Bellec, P., 2017. Resting-state network dysfunction in Alzheimer's disease: A systematic review and meta-analysis. Alzheimer's and Dementia: Diagnosis, Assessment and Disease Monitoring 8, 73-85. https://doi.org/10.1016/j.dadm.2017.03.007

Balthazar, M.L.F., de Campos, B.M., Franco, A.R., Damasceno, B.P., Cendes, F., 2014. Whole cortical and default mode network mean functional connectivity as potential biomarkers for mild Alzheimer's disease. Psychiatry Research - Neuroimaging 221, 37-42. https://doi.org/10.1016/j.pscychresns.2013.10.010

Brand, L., Nichols, K., Wang, H., Shen, L., Huang, H., 2019. Joint Multi-Modal Longitudinal Regression and Classification for Alzheimer's Disease Prediction. IEEE Transactions on Medical Imaging 14, 1-1. https://doi.org/10.1109/tmi.2019.2958943

Breukelaar, I.A., Antees, C., Grieve, S.M., Foster, S.L., Gomes, L., Williams, L.M., Korgaonkar, M.S., 2017. Cognitive control network anatomy correlates with neurocognitive behavior: A longitudinal study. Human Brain Mapping 38, 631-643. https://doi.org/10.1002/hbm.23401

Brier, M.R., Thomas, J.B., Ances, B.M., 2014. Network dysfunction in Alzheimer's disease: refining the disconnection hypothesis. Brain connectivity 4, 299-311. https://doi.org/10.1089/brain.2014.0236

Bzdok, D., Hartwigsen, G., Reid, A., Laird, A.R., Fox, P.T., Eickhoff, S.B., 2016. Left inferior parietal lobe engagement in social cognition and language. Neurosci Biobehav Rev 68, 319334. https://doi.org/10.1016/j.neubiorev.2016.02.024.Left

Calhoun, V.D., Miller, R., Pearlson, G., Adali, T., 2014. The Chronnectome: Time-Varying Connectivity Networks as the Next Frontier in fMRI Data Discovery. Neuron 84, 262-274. 
https://doi.org/10.1016/j.neuron.2014.10.015

Caspers, S., Schleicher, A., Bacha-Trams, M., Palomero-Gallagher, N., Amunts, K., Zilles, K., 2013. Organization of the human inferior parietal lobule based on receptor architectonics.

Cerebral Cortex 23, 615-628. https://doi.org/10.1093/cercor/bhs048

Cohen, M.B., Elder, S., Musco, Cameron, Musco, Christopher, Persu, M., 2015. Dimensionality reduction for k-means clustering and low rank approximation. Arxiv preprint arXiv:1410.6801. https://doi.org/10.1145/2746539.2746569

Damaraju, E., Allen, E.A., Belger, A., Ford, J.M., McEwen, S., Mathalon, D.H., Mueller, B.A., Pearlson, G.D., Potkin, S.G., Preda, A., Turner, J.A., Vaidya, J.G., Van Erp, T.G., Calhoun, V.D., 2014. Dynamic functional connectivity analysis reveals transient states of dysconnectivity in schizophrenia. NeuroImage: Clinical 5, 298-308. https://doi.org/10.1016/j.nicl.2014.07.003

Dhanjal, N.S., Wise, R.J.S., 2014. Frontoparietal cognitive control of verbal memory recall in Alzheimer's disease. Annals of Neurology 76, 241-251. https://doi.org/10.1002/ana.24199

Dong, D., Duan, M., Wang, Y., Zhang, X., Jia, X., Li, Y., Xin, F., Yao, D., Luo, C., 2019. Reconfiguration of Dynamic Functional Connectivity in Sensory and Perceptual System in Schizophrenia. Cerebral Cortex 29, 3577-3589. https://doi.org/10.1093/cercor/bhy232

Du, Y., Fu, Z., Sui, J., Gao, S., Xing, Y., Lin, D., Salman, M., Rahaman, M.A., Abrol, A., Chen, J., Hong, E., Kochunov, P., Osuch, E.A., Calhoun, V.D., 2019. NeuroMark: an adaptive independent component analysis framework for estimating reproducible and comparable fMRI biomarkers among brain disorders. medRxiv 19008631. https://doi.org/10.1101/19008631

Eliasova, I., Anderkova, L., Marecek, R., Rektorova, I., 2014. Non-invasive brain stimulation of the right inferior frontal gyrus may improve attention in early Alzheimer's disease: A pilot study. Journal of the Neurological Sciences 346, 318-322. https://doi.org/10.1016/j.jns.2014.08.036

Fiorenzato, E., Strafella, A.P., Kim, J., Schifano, R., Weis, L., Antonini, A., Biundo, R., 2019. Dynamic functional connectivity changes associated with dementia in Parkinson's disease. Brain 142, 2860-2872. https://doi.org/10.1093/brain/awz192

Fu, Z., Caprihan, A., Chen, J., Du, Y., Adair, J.C., Sui, J., Rosenberg, G.A., Calhoun, V.D., 2019. Altered static and dynamic functional network connectivity in Alzheimer's disease and subcortical ischemic vascular disease: shared and specific brain connectivity abnormalities. Human Brain Mapping 40, 3203-3221. https://doi.org/10.1002/hbm.24591

Garrity, A.G., Pearlson, G.D., Mckiernan, K., Ph, D., Lloyd, D., Ph, D., Kiehl, K.A., Ph, D., Calhoun, V.D., Ph, D., 2007. Aberrant "Default Mode" Functional Connectivity in Schizophrenia. American Journal of Psychiatry 164, 1-8. https://doi.org/10.1176/appi.ajp.164.3.450

Gaubert, S., Raimondo, F., Houot, M., Corsi, M.C., Naccache, L., Sitt, J.D., Hermann, B., Oudiette, D., Gagliardi, G., Habert, M.O., Dubois, B., De Vico Fallani, F., Bakardjian, H., Epelbaum, S., 2019. EEG evidence of compensatory mechanisms in preclinical Alzheimer's disease. Brain 142, 2096-2112. https://doi.org/10.1093/brain/awz150

Greene, S.J., Killiany, R.J., 2010. Subregions of the inferior parietal lobule are affected in the progression to Alzheimer's disease. Neurobiology of Aging 31, 1304-1311.

https://doi.org/10.1016/j.neurobiolaging.2010.04.026

Grieder, M., Wang, D.J.J., Dierks, T., Wahlund, L.-O., Jann, K., 2018. Default Mode Network Complexity and Cognitive Decline in Mild Alzheimer's Disease. Frontiers in Neuroscience 
12, 1-9. https://doi.org/10.3389/fnins.2018.00770

Gupta, Y., Lama, R.K., Kwon, G.R., 2019. Prediction and Classification of Alzheimer's Disease Based on Combined Features From Apolipoprotein-E Genotype, Cerebrospinal Fluid, MR, and FDG-PET Imaging Biomarkers. Frontiers in Computational Neuroscience 13, 1-18. https://doi.org/10.3389/fncom.2019.00072

Han, K., Chapman, S.B., Krawczyk, D.C., 2018. Neuroplasticity of cognitive control networks following cognitive training for chronic traumatic brain injury. NeuroImage: Clinical 18, 262 278. https://doi.org/10.1016/j.nicl.2018.01.030

Komarova, N.L., Thalhauser, C.J., 2011. High degree of heterogeneity in Alzheimer's disease progression patterns. PLoS Computational Biology 7.

https://doi.org/10.1371/journal.pcbi.1002251

Kruthika, K.R., Rajeswari, Maheshappa, H.D., 2019. Multistage classifier-based approach for Alzheimer's disease prediction and retrieval. Informatics in Medicine Unlocked 14, 34-42. https://doi.org/10.1016/j.imu.2018.12.003

LaMontagne, P.J., Benzinger, T.L.S., Morris, J.C., Keefe, S., Hornbeck, R., Xiong, C., Grant, E., Hassenstab, J., Moulder, K., Vlassenko, A., Raichle, M.E., Cruchaga, C., Marcus, D., 2019. OASIS-3: Longitudinal Neuroimaging, Clinical, and Cognitive Dataset for Normal Aging and Alzheimer Disease. medRxiv. https://doi.org/10.1101/2019.12.13.19014902

Lee, Garam, et al, 2019. Predicting Alzheimer's disease progression using multi-modal deep learning approach. Scientific Reports 9, 1-12. https://doi.org/10.1038/s41598-018-37769-z

Massoud, F., Léger, G.C., 2011. Pharmacological treatment of Alzheimer disease. Canadian Journal of Psychiatry 56, 579-588. https://doi.org/10.1177/070674371105601003

Masters, C.L., Bateman, R., Blennow, K., Rowe, C.C., Sperling, R.A., Cummings, J.L., 2015. Alzheimer's disease. Nature Reviews Disease Primers 1, 1-18. https://doi.org/10.1038/nrdp.2015.56

Miao, X., Wu, X., Li, R., Chen, K., Yao, L., 2011. Altered connectivity pattern of hubs in defaultmode network with alzheimer's disease: An granger causality modeling approach. PLoS ONE 6, 1-7. https://doi.org/10.1371/journal.pone.0025546

Miller, R.L., Yaesoubi, M., Turner, J.A., Mathalon, D., Preda, A., Pearlson, G., Adali, T., Calhoun, V.D., 2016. Higher Dimensional Meta-State Analysis Reveals Reduced Resting fMRI Connectivity Dynamism in Schizophrenia Patients. PloS one 11, e0149849. https://doi.org/10.1371/journal.pone.0149849

O’Bryant, S.E., Waring, S.C., Cullum, C.M., Hall, J., Lacritz, L., Massman, P.J., Lupo, P.J., Reisch, J.S., Doody, R., 2008. Staging dementia using clinical dementia rating scale sum of boxes scores: A Texas Alzheimer's research consortium study. Archives of Neurology 65, 1091-1095. https://doi.org/10.1001/archneur.65.8.1091

Oishi, A., Yamasaki, T., Tsuru, A., Minohara, M., Tobimatsu, S., 2018. Decreased gray matter volume of right inferior parietal lobule is associated with severity of mental disorientation in patients with mild cognitive impairment. Frontiers in Neurology 9, 1-5. https://doi.org/10.3389/fneur.2018.01086

Park, K.H., Noh, Y., Choi, E.J., Kim, H., Chun, S., Son, Y.D., 2017. Functional connectivity of the hippocampus in early- and vs. late-onset alzheimer's disease. Journal of Clinical Neurology (Korea) 13, 387-393. https://doi.org/10.3988/jen.2017.13.4.387

Preti, M.G., Bolton, T.A., Van De Ville, D., 2017. The dynamic functional connectome: State-ofthe-art and perspectives. NeuroImage 160, 41-54. https://doi.org/10.1016/j.neuroimage.2016.12.061 
Qiao, C., Gao, B., Lu, L.J., Calhoun, V.D., Wang, Y.P., 2019. Two-step feature selection for identifying developmental differences in resting fMRI intrinsic connectivity networks. Applied Sciences (Switzerland) 9, 1-18. https://doi.org/10.3390/app9204298

Rashid, B., Arbabshirani, M.R., Damaraju, E., Cetin, M.S., Miller, R., Pearlson, G.D., Calhoun, V.D., 2016. Classification of schizophrenia and bipolar patients using static and dynamic resting-state fMRI brain connectivity. NeuroImage 134, 645-657. https://doi.org/10.1016/j.neuroimage.2016.04.051

Roncero, C., Kniefel, H., Service, E., Thiel, A., Probst, S., Chertkow, H., 2017. Inferior parietal transcranial direct current stimulation with training improves cognition in anomic Alzheimer's disease and frontotemporal dementia. Alzheimer's and Dementia: Translational Research and Clinical Interventions 3, 247-253. https://doi.org/10.1016/j.trci.2017.03.003

Ryan, N.S., Rossor, M.N., 2011. Defining and describing the pre-dementia stages of familial Alzheimer's disease. Alzheimer's Research and Therapy 3, 2010-2012. https://doi.org/10.1186/alzrt91

Schlesinger, K.J., Turner, B.O., Grafton, S.T., Miller, M.B., Carlson, J.M., 2017. Improving resolution of dynamic communities in human brain networks through targeted node removal. PLoS ONE 12, 1-28. https://doi.org/10.1371/journal.pone.0187715

Schumacher, J., Peraza, L.R., Firbank, M., Thomas, A.J., Kaiser, M., Gallagher, P., O’Brien, J.T., Blamire, A.M., Taylor, J.P., 2019. Dynamic functional connectivity changes in dementia with Lewy bodies and Alzheimer's disease. NeuroImage: Clinical 22, 101812. https://doi.org/10.1016/j.nicl.2019.101812

Sendi, M.S.E., Pearlson, G.D., Daniel, H, J.M.F., Preda, A., Erp, T.G.M. van, D.Calhoun, V., 2020a. Multiple overlapping dynamic patterns of the visual sensory network in schizophrenia. bioRxiv. https://doi.org/https://doi.org/10.1101/2020.12.21.423535

Sendi, M.S.E., Zendehrouh, E., Fu, Z., Mahmoudi, B., Miller, R.L., Calhoun, V.D., 2020b. A Machine Learning Model for Exploring Aberrant Functional Network Connectivity Transition in Schizophrenia, in: IEEE Southwest Symposium on IEEE Southwest Symposium On. pp. $112-115$.

Shah, S.A., Koltun, V., 2017. Robust continuous clustering. Proceedings of the National Academy of Sciences of the United States of America 114, 9814-9819. https://doi.org/10.1073/pnas.1700770114

Shapiro, K., Hillstrom, A.P., 2002. Control of visuotemporal attention by inferior parietal and superior temporal cortex. Current Biology 12, 1320-1325. https://doi.org/10.1016/S09609822(02)01040-0

Shigihara, Y., Hoshi, H., Shinada, K., Okada, T., Kamada, H., 2020. Non-pharmacological treatment changes brain activity in patients with dementia. Scientific Reports 10, 1-9. https://doi.org/10.1038/s41598-020-63881-0

Sun, Y., Collinson, S.L., Suckling, J., Sim, K., 2019. Dynamic reorganization of functional connectivity reveals abnormal temporal efficiency in schizophrenia. Schizophrenia Bulletin 45, 659-669. https://doi.org/10.1093/schbul/sby077

Vergara, V.M., Mayer, A.R., Kiehl, K.A., Calhoun, V.D., 2018. Dynamic functional network connectivity discriminates mild traumatic brain injury through machine learning.

NeuroImage: Clinical 19, 30-37. https://doi.org/10.1016/j.nicl.2018.03.017

Wang, J., Xie, S., Guo, X., Becker, B., Fox, P.T., Eickhoff, S.B., Jiang, T., 2017. Correspondent Functional Topography of the Human Left Inferior Parietal Lobule at Rest and Under Task Revealed Using Resting-State fMRI and Coactivation Based Parcellation. Human Brain 
Mapping. https://doi.org/10.1002/hbm.23488

Wang, K., Liang, M., Wang, L., Tian, L., Zhang, X., Li, K., Jiang, T., 2007. Altered functional connectivity in early Alzheimer's disease: A resting-state fMRI study. Human Brain Mapping 28, 967-978. https://doi.org/10.1002/hbm.20324

Wang, Y., Qin, Y., Li, H., Yao, D., Sun, B., Li, Z., Li, X., Dai, Y., Wen, C., Zhang, L., Zhang, C., Zhu, T., Luo, C., 2019. Abnormal Functional Connectivity in Cognitive Control Network, Default Mode Network, and Visual Attention Network in Internet Addiction: A Resting-State fMRI Study. Frontiers in Neurology 10. https://doi.org/10.3389/fneur.2019.01006

Wang, Z., Qiao, K., Chen, G., Sui, D., Dong, H.M., Wang, Y.S., Li, H.J., Lu, J., Zuo, X.N., Han, Y., 2019. Functional connectivity changes across the spectrum of subjective cognitive decline, amnestic mild cognitive impairment and alzheimer's disease. Frontiers in Neuroinformatics 13, 1-11. https://doi.org/10.3389/fninf.2019.00026

Wang, Z., Xia, M., Dai, Z., Liang, X., Song, H., He, Y., Li, K., 2015. Differentially disrupted functional connectivity of the subregions of the inferior parietal lobule in Alzheimer's disease. Brain Structure and Function 220, 745-762. https://doi.org/10.1007/s00429-013-0681-9

Westerhausen, R., Moosmann, M., Alho, K., Belsby, S.O., Hämäläinen, H., Medvedev, S., Specht, K., Hugdahl, K., 2010. Identification of attention and cognitive control networks in a parametric auditory fMRI study. Neuropsychologia 48, 2075-2081. https://doi.org/10.1016/j.neuropsychologia.2010.03.028

Woodard, J.L., Grafton, S.T., Votaw, J.R., Green, R.C., Dobraski, M.E., Hoffman, J.M., 1998. Compensatory recruitment of neural resources during overt rehearsal of word lists in Alzheimer's disease. Neuropsychology 12, 491-504. https://doi.org/10.1037/08944105.12.4.491

Yetkin, F.Z., Rosenberg, R.N., Weiner, M.F., Purdy, P.D., Cullum, C.M., 2006. FMRI of working memory in patients with mild cognitive impairment and probable Alzheimer's disease. European Radiology 16, 193-206. https://doi.org/10.1007/s00330-005-2794-X

Yiannopoulou, K.G., Papageorgiou, S.G., 2020. Current and Future Treatments in Alzheimer Disease: An Update. Journal of Central Nervous System Disease 12, 117957352090739. https://doi.org/10.1177/1179573520907397

Yoav Benjamini $\square$; Yosef Hochberg, 1995. Controlling the False Discovery Rate $\square$ : A Practical and Powerful Approach to Multiple Testing. Royal Statistical Society . Series B ( Methodological ) 57, 289-300.

Zendehrouh, E., Sendi, M.S.E., Sui, J., Fu, Z., Zhi, D., Lv, L., Ma, X., Ke, Q., Li, X., 2020. Aberrant Functional Network Connectivity Transition Probability in Major Depressive Disorder, in: 42nd Annual International Conference of the IEEE Engineering in Medicine \& Biology Society (EMBC). pp. 1493-1496.

Zhao, J., Du, Y.H., Ding, X.T., Wang, X.H., Men, G.Z., 2020. Alteration of functional connectivity in patients with Alzheimer's disease revealed by resting-state functional magnetic resonance imaging. Neural Regeneration Research 15, 285-292. https://doi.org/10.4103/16735374.265566

Zhi, D., Calhoun, V.D., Lv, L., Ma, X., Ke, Q., Fu, Z., Du, Y., Yang, Y., Yang, X., Pan, M., Qi, S., Jiang, R., Yu, Q., Sui, J., 2018. Aberrant Dynamic Functional Network Connectivity and Graph Properties in Major Depressive Disorder. Frontiers in Psychiatry 9, 1-11. https://doi.org/10.3389/fpsyt.2018.00339

Zhong, Y., Huang, L., Cai, S., Zhang, Y., von Deneen, K.M., Ren, A., Ren, J., 2014. Altered effective connectivity patterns of the default mode network in Alzheimer's disease: An fMRI 
study. Neuroscience Letters 578, 171-175. https://doi.org/10.1016/j.neulet.2014.06.043

Zucchella, C., Sinforiani, E., Tamburin, S., Federico, A., Mantovani, E., Bernini, S., Casale, R.,

Bartolo, M., 2018. The Multidisciplinary Approach to Alzheimer's Disease and Dementia. A Narrative Review of Non-Pharmacological Treatment. Frontiers in Neurology 9, 1-22.

https://doi.org/10.3389/fneur.2018.01058 
Table 1. Demographic and clinical information

\begin{tabular}{|c|lc|}
\hline \multirow{5}{*}{ HC } & N & 1028 \\
& Gender $(\mathrm{M} / \mathrm{F})$ & $415 / 613$ \\
& Age & $69.83 \pm 8.64$ \\
& CDR-SOB & $0 \pm 0$ \\
& MMSE & $28.88 \pm 1.34$ \\
\hline \multirow{3}{*}{ vmAD } & N & 277 \\
& Age & $215 / 142$ \\
& CDR-SOB & $75.18 \pm 7.77$ \\
& MMSE & $2.59 \pm 1.92$ \\
& MMS) & $25.94 \pm 3.60$ \\
\hline
\end{tabular}

Note: HC: Healthy control ; vMAD: very mild Alzheimer's disease; M:male; F:female; CDR-SOB: clinical dementia rating scale sum of boxes, MMSE: mini-mental state examination 
Table 2. Component Labels

\begin{tabular}{|l|ccc|}
\hline \multicolumn{1}{|c|}{ Component name } & \multicolumn{3}{c|}{ Peak coordinate (mm) } \\
\hline (IC 68) Inferior parietal lobule [IPL] & -30.5 & -61.5 & 43.5 \\
(IC 33) Insula & -0.5 & 22.5 & -3.5 \\
(IC 43) Superior medial frontal gyrus [SMFG] & -48.5 & 34.5 & 29.5 \\
(IC 70) Inferior frontal gyrus [IFG] & 53.5 & 22.5 & -0.5 \\
(IC 61) Right inferior frontal gyrus [R IFG] & -41.5 & 19.5 & 13.5 \\
(IC 55) Middle frontal gyrus [MiFG] & -53.5 & -49.5 & 43.5 \\
(IC 63) Inferior parietal lobule [IPL] & 44.5 & -34.5 & 46.5 \\
(IC 79) Left inferior parietal lobue [L IPL] & -6.5 & 13.5 & 64.5 \\
(IC 84) Supplementary motor area [SMA] & -24.5 & 26.5 & 49.5 \\
(IC 96) Superior frontal gyrus [SFG] & 30.5 & 41.5 & 28.5 \\
(IC 88) Middle frontal gyrus [MiFG] & 23.5 & -9.5 & -16.5 \\
(IC 48) Hippocampus [HiPP] & 45.5 & -61.5 & 43.5 \\
(IC 81) Left inferior parietal lobue [L IPL] & -15.5 & 20.5 & 37.5 \\
(IC 37) Middle cingulate cortex [MCC] & 39.5 & 44.5 & -0.5 \\
(IC 67) Inferior frontal gyrus [IFG] & -26.5 & 47.5 & 5.5 \\
(IC 38) Middle frontal gyrus [MiFG] & -24.5 & -36.5 & 1.5 \\
(IC 83) Hippocampus [HiPP] & & &
\end{tabular}




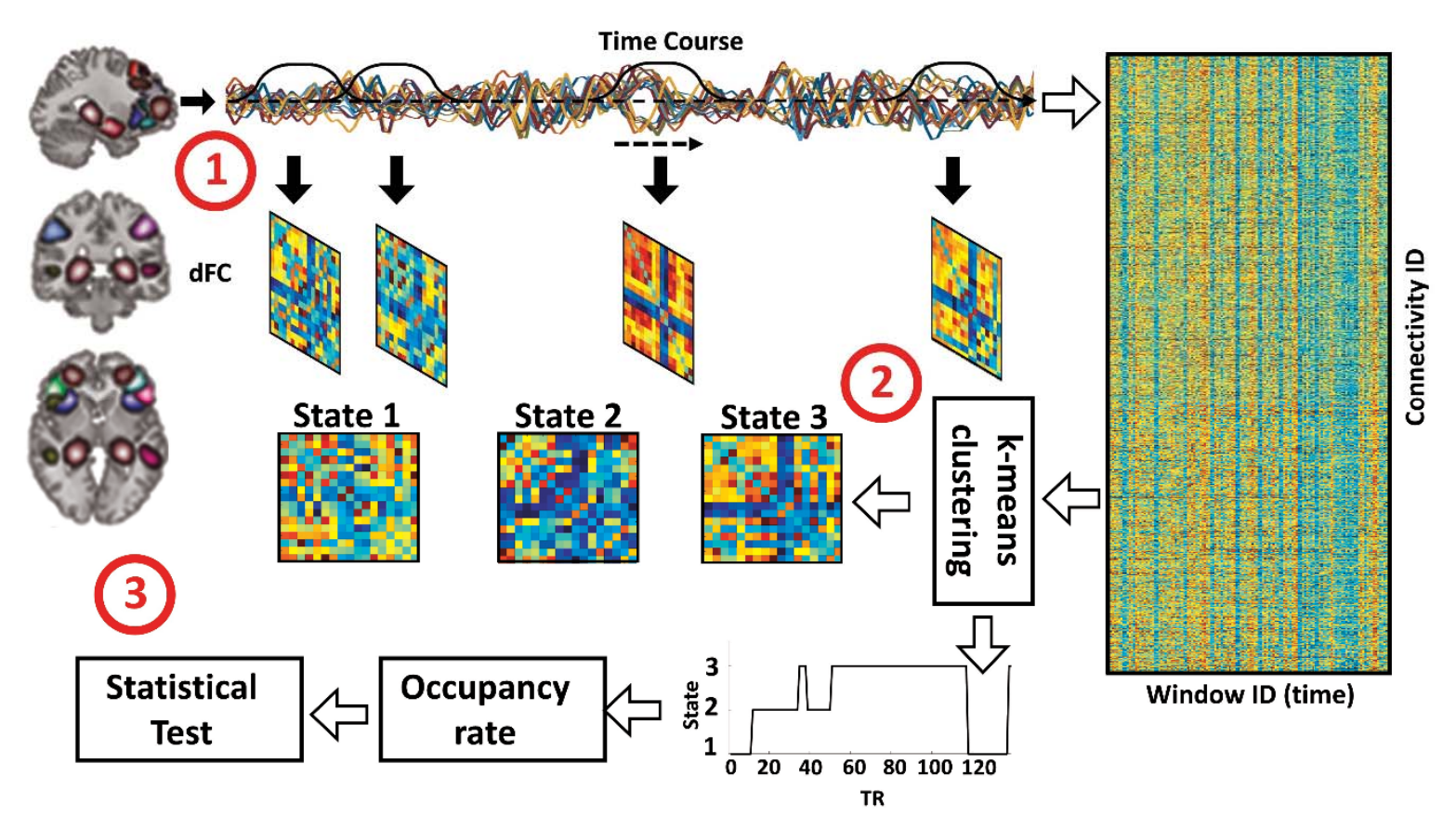

Fig. 1. Analytic pipeline. Step1: The time-course signal of 17 components in the cognitive control network $(\mathrm{CCN})$ has been identified using group-ICA. After identifying 17 regions in $\mathrm{CCN}$, a taper sliding window was used to segment the time-course signals and then calculated the functional connectivity (FC). Step2: After vectorizing the FC matrixes, we have concatenated them, and then a k-means clustering, $\mathrm{k}=3$, was used to group FCs to three distinct states. Elbow criteria were used to find the optimal k. In addition, the Euclidean distance metric is used in this clustering. Step3: Then, based on the state vector of each subject, the occupancy rate or OCR features, in total 3 features, were calculated from the state vector of each subject. Then, we explored the link between the state-specific connectivity feature and the clinical rate. In addition, we investigated the link between OCR with the clinical rate. 
State 1

$33.68 \%$

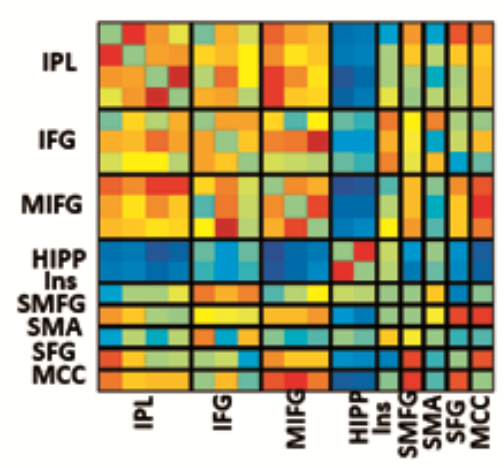

State 2

28.02\%

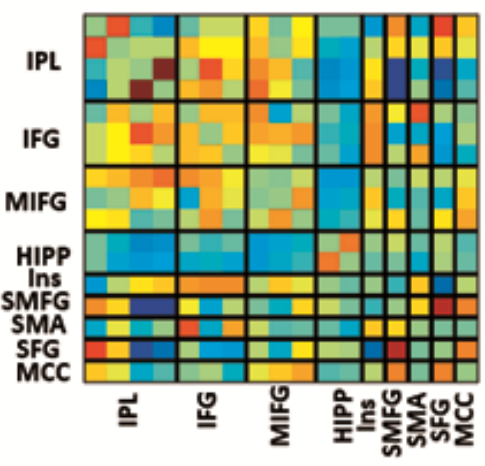

State 3

$38.30 \%$

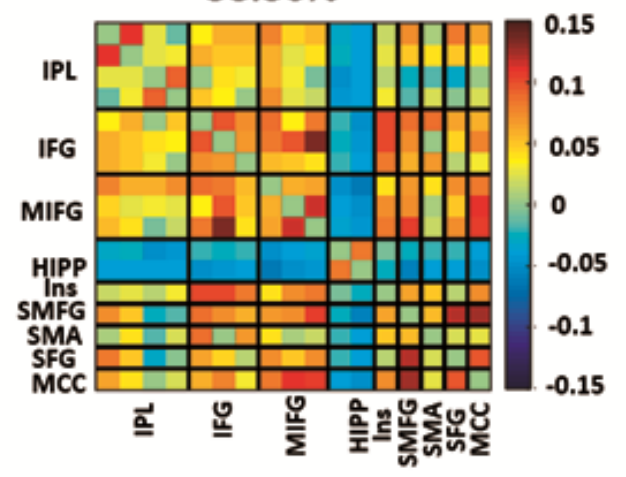

Fig.2. Dynamic functional connectivity (dFC) states. The three identified dFC states using the k-means clustering method. Each state is a $17 \times 17$ matrix in which the positive connectivity is shown with hot, and the negative connectivity is shown in cold color. We put all 17 components in 9 regions including inferior parietal lobule (IPL), inferior frontal gyrus (IFG), middle frontal gyrus (MIFG) hippocampus (HIPP), insula (INS), Superior medial frontal gyrus (SMFG), supplementary motor area (SMA), superior frontal gyrus(SFG), and middle cingulate cortex (MCC). In all states, the hippocampus showed a connection with the rest of the CCN. However, the connectivity between the hippocampus and the rest of the $\mathrm{CCN}$ in higher in state 2. Also, state 3 showed the strongest positive connectivity within the inferior frontal gyrus. We found subjects spend $33.68 \%, 28.02 \%$, and $38.30 \%$ in state 1 , state 2 , and state 3 , respectively. 

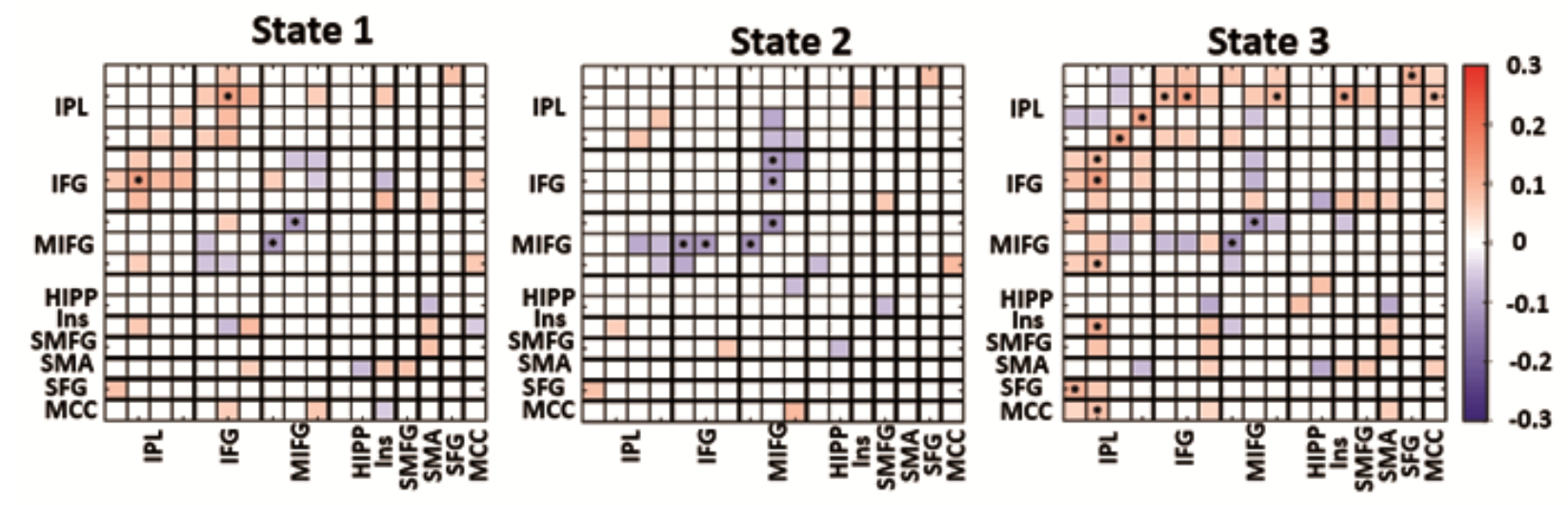

Fig. 3: Correlation between FC of each state and MMSE. In each state, we averaged the cell features of all $\mathrm{dFC}$ of each subject, and then we calculated the partial correlation between averaged cell features of each subject and MMSE by controlling the age and gender to explore how these features changed by progressing from normal stage to AD stages. The correlation with significant $\mathrm{p}$ value $(\mathrm{p} \leq 0.05)$ is highlighted in red and blue. A significant group difference that passes the multiple comparisons is marked by asterisks (false discovery rate [FDR] corrected, $\mathrm{q}=0.05)$. Inferior parietal lobule (IPL), inferior frontal gyrus (IFG), middle frontal gyrus (MIFG) hippocampus (HIPP), insula (INS), Superior medial frontal gyrus (SMFG), supplementary motor area (SMA), superior frontal gyrus(SFG), and middle cingulate cortex

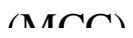




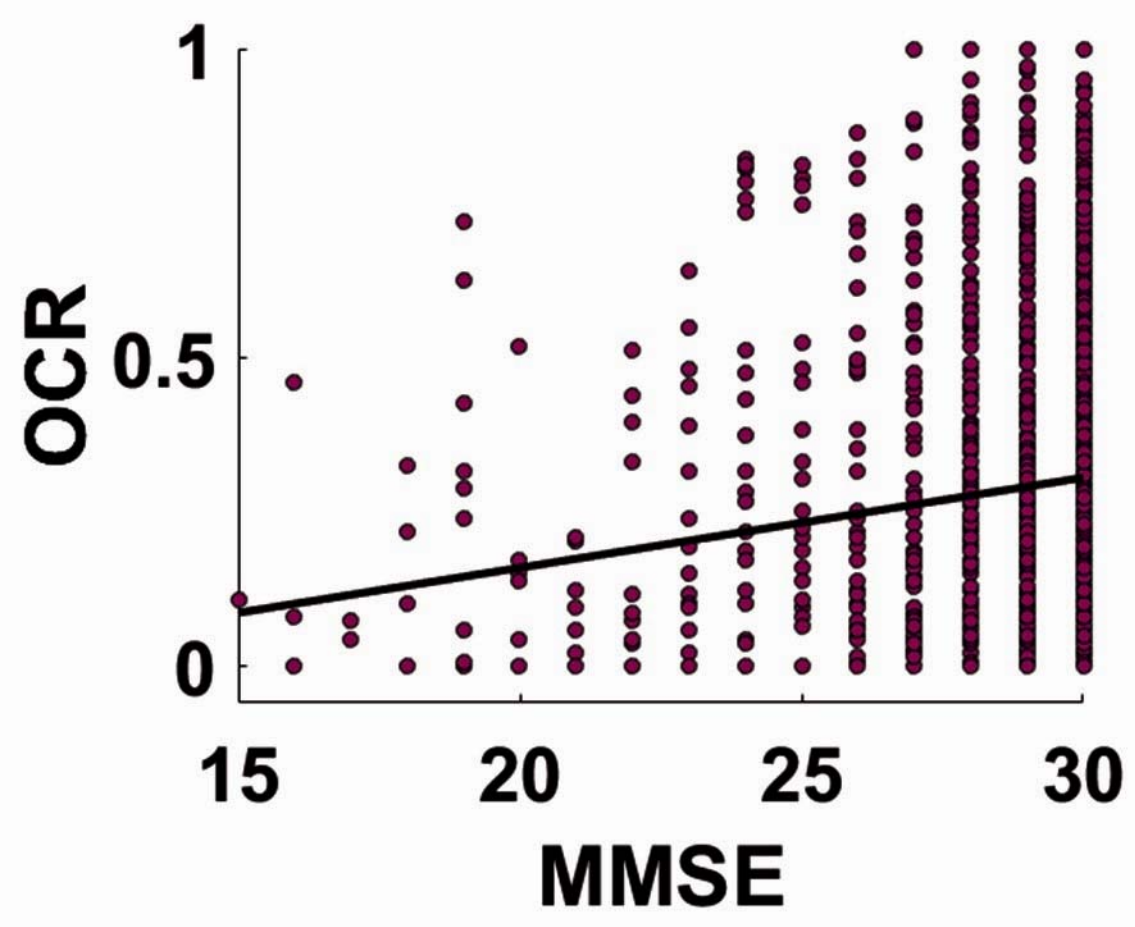

Fig. 4: Correlation between OCR and MMSE. The partial correlation between the occupancy rate of state 2 with MMSE. $r=0.073$, FDR corrected $\mathrm{p}=0.01, \mathrm{n}=1385$. 